\title{
Star formation thresholds derived From THINGS
}

\author{
F. Bigiel ${ }^{1}$, F. Walter ${ }^{1}$, E. de Blok ${ }^{2}$, E. Brinks ${ }^{3}$ and B. Madore ${ }^{4}$ \\ ${ }^{1}$ Max-Planck Institut für Astronomie, Königstuhl 17, 69117, Heidelberg, Germany \\ ${ }^{2}$ Research School of Astronomy \& Astrophysics, Mount Stromlo Observatory, Cotter Road, \\ 2611, Weston, Australia \\ ${ }^{3}$ Centre for Astrophysics Research, Science \& Technology Research Institute, University of \\ Hertfordshire, AL10 9AB, Hatfield, UK \\ ${ }^{4}$ Observatories of the Carnegie Institution of Washington, 813 Santa Barbara St., 91101, \\ Pasadena, USA
}

\begin{abstract}
We present first results from THINGS (The HI Nearby Galaxy Survey), which consists of high quality HI maps obtained with the VLA of 34 galaxies across a wide range of galaxy parameters (Hubble type, mass/luminosity). We compare the distribution of HI to the UV emission in our sample galaxies. In particular we present radial profiles of the HI (tracing the neutral interstellar medium) and UV (mainly tracing regions of recent star formation) in our sample galaxies. The azimuthally averaged HI profiles are compared to the predicted critical density above which organized large-scale star formation is believed to start (this threshold is based on the Toomre-Q parameter, which in turn is a measure for local gravitational instability).
\end{abstract}

Keywords. galaxies: ISM, radio lines: ISM, galaxies: evolution

\section{Main}

The HI Nearby Galaxy Survey (THINGS) provides high-resolution maps of the $21 \mathrm{~cm}$ line of atomic hydrogen (HI) at high spatial resolution (6") of a sample of nearby galaxies. This resolution is very well matched to the resolution of GALEX, the Galaxy Evolution Explorer, which operates in the ultraviolet regime. In a first analysis, we compare the azimuthally averaged HI column densities to the UV profiles in our sample galaxies (the deprojection parameters have been derived from a tilted ring analysis of the targets). To first order, the UV emission traces the distribution of the HI well in the centers of galaxies: The UV emission typically picks up at radii where the HI column density exceeds the critical density for star formation as given by either a constant density threshold of $\sim 7 \times 10^{20} \mathrm{~cm}^{-2}$, or, for galaxies where rotation curves could be derived, as given by a radius dependent critical density as calculated from the Toomre-Q parameter (this parameter is related to local gravitational instabilities in the gas and can be used to derive a column density which is believed to be critical for those instabilities to collapse). Comparisons of HI column density maps with maps of UV emission show hints of faint UV emission reaching far out to large galactocentric radii, i.e. well out to the extended HI disks. Pixel-by-pixel plots of HI- and UV emission show that the UV emission is, to first order, a function of HI flux in more massive galaxies. Our unique dataset will allow us to search for trends in the relation between HI and UV as a function of environment and galaxy type. 